\title{
Active optics methods for exoplanet direct imaging (Research Note)
}

\section{Stress polishing of supersmooth aspherics for VLT-SPHERE planet finder}

\author{
E. Hugot ${ }^{1}$, M. Ferrari ${ }^{1}$, K. El Hadi ${ }^{1}$, A. Costille ${ }^{2}$ K. Dohlen ${ }^{1}$, P. Rabou ${ }^{2}$, P. Puget ${ }^{2}$, and J. L. Beuzit ${ }^{2}$ \\ ${ }^{1}$ Laboratoire d'Astrophysique de Marseille, Centre National de la Recherche Scientifique (CNRS/INSU), Aix-Marseille Université, \\ LAM, 38 rue Frederic Joliot Curie, 13388 Marseille Cedex 13, France \\ e-mail: emmanuel.hugot@oamp.fr \\ 2 UJF-Grenoble 1/CNRS-INSU, Institut de Planétologie et d'Astrophysique de Grenoble (IPAG) UMR 5274, 38041 Grenoble, France
}

Received 3 August 2011 / Accepted 30 November 2011

\begin{abstract}
Context. The next generation of exoplanet hunters will be targeting hot Jupiter-like exoplanets orbiting around nearby stars through direct imaging. The high contrast needed for such planet finders requires optical surfaces free of high spatial frequency ripples that might remain in the post-coronagraphic image as quasi-static speckles.

Aims. We report results on the manufacturing of three supersmooth aspherical mirrors for the ESO/VLT-SPHERE instrument. The excellent optical quality obtained will allow the future planet hunter to increase the level of achievable contrast by a strong reduction of the noise level and residual quasi-static speckles on the image plane.

Methods. The stress polishing method used on these mirrors is well suited to superpolishing aspheric components for astronomy. The main advantage of this technique is the very high optical quality obtained either on the form errors or on the high spatial frequency errors. Furthermore, the roughness can be decreased to a few angstroms, thanks to the classical polishing with a large pitch tool.

Results. Interferograms obtained during polishing and in the final stage show the supersmooth quality of each mirror. Errors in the high spatial frequency range are lower than $4 \mathrm{~nm} \mathrm{rms} \mathrm{WF}$, thereby avoiding the degradation of the post-coronagraphic image. A comparison of the power spectral density (PSD) obtained by stress polishing and the PSD of the VLT-UT3 primary mirror demonstrates that the contrast capabilities will not be limited by the aspherical mirrors.
\end{abstract}

Key words. instrumentation: adaptive optics - instrumentation: high angular resolution

\section{Exoplanet direct imaging}

The search for extrasolar planets is now one of the main exciting fields in astronomy ever since the discovery of 51Peg by Mayor \& Queloz (1995) using radial velocity methods (Baranne et al. 1996). Indirect detection methods have been under developement for years, but direct imaging from the ground is now a concrete challenge that will be tackled by the future generation of adaptive optics (AO) assisted planet hunters on $8 \mathrm{~m}$-class telescopes, such as ESO/VLT-SPHERE ${ }^{1}$ (Beuzit et al. 2010) and Gemini Planet Imager (GPI, Macintosh et al. 2006).

Direct imaging of faint companion close to a bright star is a hard task requiring a specific instrumentation, such as eXtreme Adaptive Optics (XAO) for correcting the atmospheric turbulence of ground based instrumentation (Fusco et al. 2006) and coronagraphic devices for the extinction of the starlight to increase the contrast (Roddier \& Roddier 1997; Rouan et al. 2000; Soummer et al. 2003; N'Diaye et al. 2010). These specific techniques, combined with post processing, will allow reaching very high contrasts up to $10^{-6}$ in $J, H, K$ bands on this next generation of high dynamic instruments.

In this context, the main limitations in high-contrast coronagraphic images come from the residual quasi-static speckles (Soummer et al. 2007) induced by the atmospheric phase

\footnotetext{
${ }^{1}$ SPHERE stands for Spectro-Polarimetric High contrast imaging for Exoplanet REsearch.
}

residuals and instrumental static and quasi-static aberrations not corrected by AO. While AO residuals evolve quickly and average out with time, static and quasi-static speckles remain in the corrected image, with a coherence time extending from tens of seconds to several minutes (Macintosh et al. 2005; Hinkley et al. 2007). Strategies for reducing speckle noise, such as differential imaging using the wavelength dependance of speckles, have been proposed by Racine et al. (1999), and extensive simulations made by Vigan et al. (2010) show a substantial gain in contrast, typically 1.5 to 2.5 mag using a combination of spectral and angular differential imaging. However, the final performance depends on the level of noise obtained on the images. Indeed, the post-coronagraphic image quality is directly linked to the power spectral density (PSD) of the optical train before the coronagraph, equal the the squared module of the total wavefront error Fourier transform. In order to increase this image quality, a strong effort must be made at the core of instruments: the quality of optical surfaces must be increased specifically in the range of spatial frequencies not corrected by the AO module. These so-called high spatial frequency errors $(\mathrm{HiF})$ will remain in the field of view as quasi-static speckles: minimizing these errors at the polishing stage will directly affect the final contrast obtained outside the AO-corrected radius on the image. Furthermore, HiF errors can also scatter light into the dark hole though "frequency folding" (2006) and, in addition, can degrade final contrast through Fresnel propagation and chromatic beam 
shear (2008). The second parameter, roughness, will affect the final Strehl ratio obtained by the XAO system by a reduction of the amount of scattered light.

\section{Supersmooth aspherical mirrors for high-contrast capabilities}

Our purpose here is to develop a manufacturing method that increases the optical quality of aspherical surfaces which are often use in optical designs. To reach this goal, we propose a combination of two methods. Stress polishing and superpolishing techniques can be combined in a simple way, resulting in a substantial gain on each parameter that defines an optical surface and affects the final contrast in coronagraphic images.

The polishing of optical surfaces is a delicate operation, the basics of which are given in several books (Texereau 1948; Ingalls 1937). Either the sphere or the plane is the natural surface generated by the friction of two solids of similar dimensions in a relative motion with three degrees of freedom. However, the use of aspherical surfaces is useful in many optical instruments and telescopes, but their fabrication is time-consuming and costly. The surfacing of aspherical surfaces has motivated several developments since the 1930's. The German astronomer Bernhard Schmidt, pioneer in Active Optics, built the entrance corrective plate of his wide-field telescope by figuring the plate under pressure with a full-size spherical tool (Schmidt 1932). Although Schmidt just made experiments, André Couder explained this technique with elasticity equations (Couder 1940).

During the past few years, research has been pursued to solve the problem of figuring an aspherical surface of optical quality. Various modern techniques are based on variable thickness coatings, ion beam figuring, petal tools, diamond turning, or numerically controlled polishing (Marioge 1984). Further developments in magneto-rheologic polishing have allowed the production of quasi-perfect surfaces of small asphericities (Jacob 1999). These surfacing techniques are extremely powerful for generating the aspherical optical components with high optical quality but are limited by slope errors that generate HiF ripples, whose diffracting effects will degrade the resulting image quality. Nowadays, strong efforts are being made by the optical fabrication community to solve this problems, with algorithm optimisations and new materials for the tools (Dae Wok Kim 2010). However, these intrinsic limitations to all these techniques are drawbacks, especially in the field of high-contrast imaging.

Manufacturing aspherical optics with stress polishing is a mature technique (Lemaitre 2009). Its principle relies on spherical polishing with a full-sized lap of a warped substrate, which becomes aspherical once unwarped. The main advantage of this approach is the very high optical quality obtained in terms of both form and HiF errors. Achieving aspherical optics under stress requires minimizing both warping and polishing errors. The correct warping function, corresponding to the exact inverse of the final aspherical shape is obtained using analytical calculations based on elasticity theory. A specific blank geometry and load configuration is defined (Lemaitre 1972), and finite-element analysis (FEA) then allows optimization of the blank shape to increase the optical quality of the warping function. Warping the substrate during polishing allows using a full sized lap, avoiding the generation of classical sub-aperture tool marks, but also allows simple superpolishing to get supersmooth surfaces.

Superpolishing means the ability to decrease the roughness of polished surfaces by optimising each parameter of the polishing process, such as the type of lap, the polishing slurry, the temperature, speed, and time of a polishing run. Leistner et al. (1992) extensively analysed supersmooth flat surfaces using teflon or pitch laps on several materials with several polishing powders. However, the study was limited to flat surfaces because of the manufacturing process of the teflon lap (Leistner 1993). Tin laps are also used for the superpolishing of sapphire, reaching less than $1 \AA$ roughness (Weiss 1992). Our interest here is to focus on the superpolishing of Zerodur: according to the Leistner study, roughness can be decreased down to less than $2 \AA$, using a pitch lap with aluminium oxide $\left(\mathrm{Al}_{2} \mathrm{O}_{3}\right)$ in a colloidal slurry.

The combination of these two powerful techniques has been implemented for manufacturing the three toric mirrors for the VLT SPHERE instrument, which is described in the next section.

\section{VLT-SPHERE toric mirrors}

The SPHERE second generation instrument for the VLT aims at imaging Jupiter-like exoplanets orbiting a nearby star ( 100 parsecs) by using a combination of innovative optical technologies and image processing. The XAO module $\mathrm{SAXO}^{2}$ must be able to reach a strehl ratio of $90 \%$ in the $\mathrm{H}$ band $(1.65 \mu \mathrm{m})$ by correcting the atmospheric turbulence to up to 1300 eigenmodes with a high-order deformable mirror (HODM) controlled by $41^{2}$ actuators. To reach this tight performance, a very strict error budget is necessary on relay optics, especially aspherical ones.

Three toricmMirrors (TMs) are placed in the AO common path optical train to relay the beam to and from the HODM and to correct for aberrations of the off-axis design. The optical train was specifically designed to use stress-polished aspherical mirrors. This approach has allowed a significant reduction in the size of the optical system and the reduction of the number of aspherics from four off-axis parabolas to three spherically polished TMs. These toroidal optics offer the possibility of aspherical correction without introducing local surface errors thanks to the stress polishing technique used for their production.

The SPHERE instrument requires extreme optical qualities for aspherical optics, specifically in terms of local errors and roughness, in order to reach the high contrast needed for exoplanet direct imaging. Indeed, these defects are not corrected by the HODM and will remain in the image plane as residual quasi-static speckles, limiting the final contrast capabilities. This limitation due to the HODM capabilities allowed defining spatial frequency ranges in order to specify the expected optical quality on TMs. The border frequencies correspond to the upper limit of $\mathrm{AO}$ calibration and the cutoff frequency. Calibration allows correction of fixed low-frequency aberrations in the differential paths between science and wave front sensor channels (non-common path aberrations). The dynamic range of this calibration is given by the capacity of the wave-front sensor to work with a nonzero aberration level. For SPHERE, the low-frequency range (LoF) upper limit was set to four cycles per pupil (c/p), with an amount of allowed error at $50 \mathrm{~nm}$ rms on the wave-front (WF). The mid frequency range (MiF) was defined between $4 \mathrm{c} / \mathrm{p}$ and $20 \mathrm{c} / \mathrm{p}$ with an amount of allowed surface error at $10 \mathrm{~nm} \mathrm{rms}$ WF. Residual errors with a frequency higher than $20 \mathrm{c} / \mathrm{p}$ are considered to be in the HiF range, and must be lower than $6.0 \mathrm{~nm}$ rms WF.

The three toric mirrors part were completed in March 2011 and delivered to the VLT-SPHERE instrument. The stress polishing process used for their production, based on elasticity theory,

${ }^{2}$ SAXO stands for SPHERE AO for eXtreme Observations. 


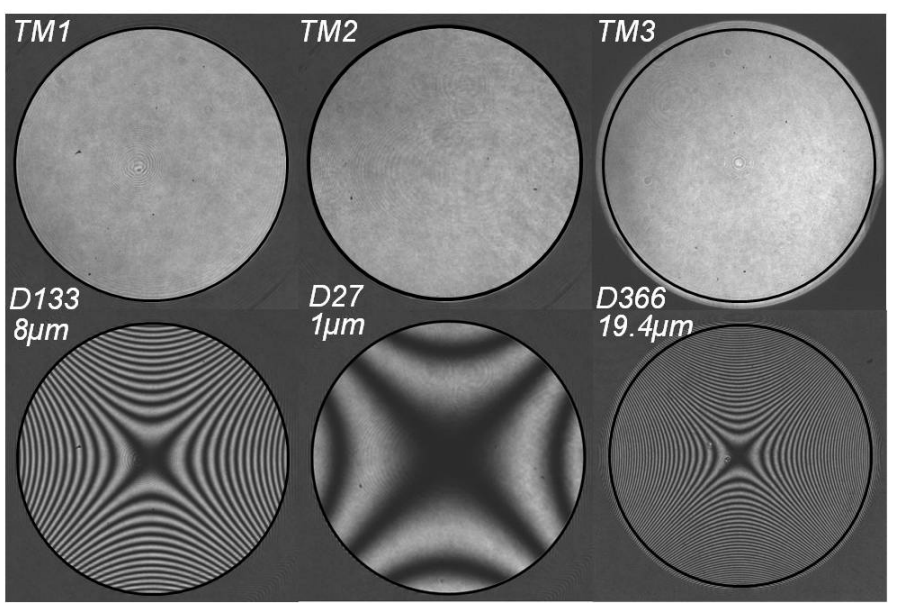

Fig. 1. He-Ne interferograms obtained with Fizeau interferometer for TM1, 2, and 3 delivered for the SPHERE instrument at the end of the spherical polishing (top) and after removal of the loads (bottom), showing the excellent optical quality obtained on peak-to-valley deformations of $8 \mu \mathrm{m}, 1 \mu \mathrm{m}$, and $19.4 \mu \mathrm{m}$. Results are listed in Table 1 .

has been described completely in our latest articles (Hugot et al. 2008, 2009). A toric surface with small aperture number and small asphericity can be described as a combination of a perfect sphere plus a pure third-order astigmatism. From that, the warping function of the mirror was optimised to generate this astigmatism with a minimal amount of residuals. This can be achieved by applying two pairs of equal and opposite forces at the end of two orthogonal diameters of the Zerodur substrate. To avoid generating astigmatism harmonics and local errors, the shape of the substrate was optimised using an angular thickness distribution, allowing a smooth transmission of stress within the blank. This specific shape, obtained from elasticity equations (Timoshenko 1959), was optimised using finite element analysis and the the mechanical displacement of the optical surface was characterised using an interferometric software. This specific geometry was directly machined onto the back of the Zerodur blank prior to the polishing process.

\section{Polishing and interferometric results}

Results in terms of surface quality are shown by the interferograms in Fig. 1, obtained with a Fizeau interferometer whose precision is better than $3 \mathrm{~nm}$ rms, with an accuracy better than $1 \mathrm{~nm} \mathrm{rms}$. Top interferograms show the optical quality at the end of the spherical polishing, clearly displaying that no structured $\mathrm{MiF}$ or HiF errors were generated during the process. This result is confirmed by the lower interferograms that show the surface deviation after removal of the loads (i.e. blank at rest). The regularity of fringes is the guaranty of the high-quality spherical polishing. From these interferograms, phase-shifting interferometry allowed computing phase maps. On the average, 36 measurements were performed for each mirror to reduce by a factor of 6 the random errors introduced by the test set-up.

From these OPD maps, we compared the power spectral density (PSD) of the three TMs with the PSD of the VLT-UT3 primary mirror. We chose to consider an azimuthal average of the 2D-PSD, defined as the squared modulus of the wavefront error Fourier transform $\left(P S D=|T F(W)|^{2}\right)$. This function is directly related to the noise level in the coronagraphic image. According to Walsh et al. (1999), PSD can be considered as a descriptor of stochastic aspects of the polishing process, while form errors

\begin{tabular}{|lll|}
\hline - VLT M1 UT3 & -TM1 SPHERE & TM2 SPHERE \\
— TM3 SPHERE & - S-TM3 SPHERE & - OAP/sub aperture tool \\
\hline
\end{tabular}

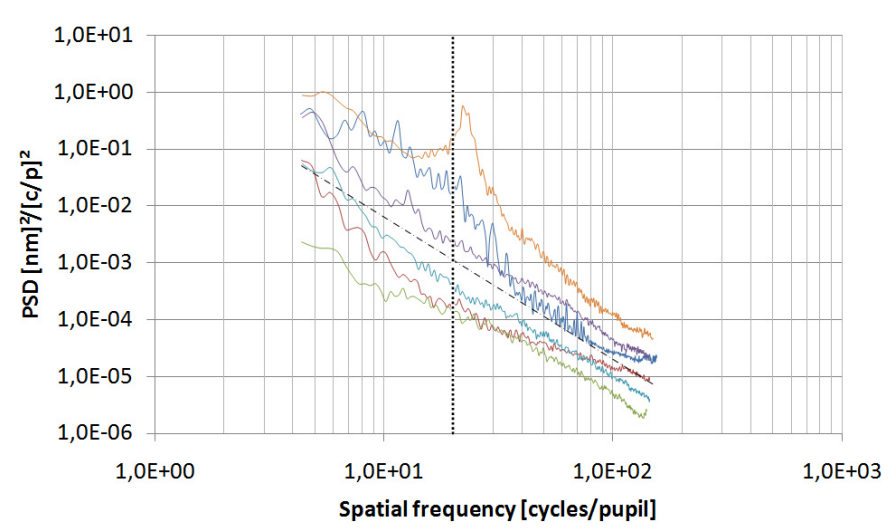

Fig. 2. 2D-power spectral density azimuthal averages. Comparison between the VLT UT3 primary mirror M1, the three TMs, and a subaperture tool polished off-axis parabola with a peak corresponding to the spatial frequency of the tool. The dashed line indicates an $f^{-2.5}$ PSD. The vertical dotted line indicates the $\mathrm{AO}$ corrected radius, showing that the high-contrast capacities of the instrument will not be limited by the TMs.

are described better by conventional aberration functions such as Seidel or Zernike polynomials. For that reason, the PSD calculation is improved by subtracting low-order surface errors prior to the data analysis (up to $4 \mathrm{c} / \mathrm{p}$ in the case of SPHERE). Figure 2 plots the results obtained and the limit of the AO-corrected radius. The first qualitative observation shows that each TM PSD follows a power law between $f^{-1.5}$ and $f^{-2.5}$, classically obtained by spherical polishing, while the telescope primary has a concentration of power in the MiF range and the beginning of the $\mathrm{HiF}$ range. This PSD behaviour is characteristic of the polishing process using sub-aperture tools, mandatory for such a large piece. However, a large amount of MiF errors will be corrected by the XAO module, leaving only HiF errors. The final contrast obtained in the coronagraphic image will not be limited by the TMs but by the telescope itself.

Integrating the PSD between two frequency limits $\left[f_{1} ; f_{2}\right]$ gives the rms wave front error denoted $\sigma$ in this specific window. This operation allows calculating residual errors while loworder terms and form errors are evaluated from the OPD map. Results summarised in Table 1 demonstrate the powerful capacity of stress polishing for producing smooth mirrors exempt from random errors or structured errors classically due to sub-aperture tool marks. The reflected wavefront quality (twice the surface error) is much better than expected: less than $\lambda / 15$ on form errors and less than $\lambda / 150$ on $\operatorname{MiF}$ and $\operatorname{HiF}(\lambda=632.8 \mathrm{~nm})$.

Furthermore the superpolishing technique using the full sized pitch tool allowed decreasing the roughness down to $0.5 \mathrm{~nm} \mathrm{rms}$ for TM1, $0.2 \mathrm{~nm} \mathrm{rms}$ for TM2, and $0.9 \mathrm{~nm} \mathrm{rms}$ for TM3. Roughness was measured on a window of $200 \mu \mathrm{m}^{2}$ with a resolution of $1 \mu \mathrm{m}$. This impressive result was obtained after a finishing run of only 30 min using $\mathrm{Al}_{2} \mathrm{O}_{3}$ polishing agent, and it is almost easy to manage thanks to the simple spherical polishing.

Finally, the three TMs have been delivered and installed on the SPHERE bench (Fig. 3). Measurements were performed on focus using a $32 \times 32$ subaperture Shack Hartman wave front sensor, allowing the alignments to be optimised, and it quantified the final form errors introduced by the three TMs and the three flat-folding mirrors placed in the common path. The measured error of $68 \mathrm{~nm} \mathrm{rms} \mathrm{WF} \pm 3$ is fully consistent with the expected 


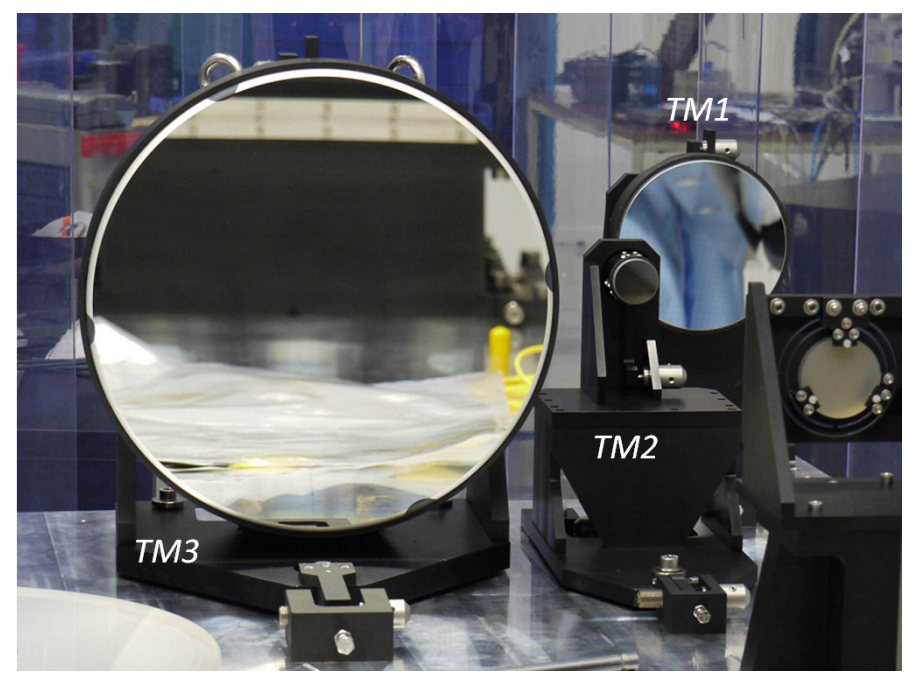

Fig. 3. View of the three TMs coated and mounted on the SPHERE optical bench.

Table 1. Final results for the three SPHERE-TMs.

\begin{tabular}{lcccc}
\hline \hline & TM1 & TM2 & TM3 & VLT M1 \\
\hline Diameter [mm] & 133 & 27 & 366 & 8000 \\
Focal ratio & F/6 & F/15 & F/5 & \\
Astm3 rms [nm] & 1651.2 & 214.4 & 3962.3 & - \\
LoF WFE [nm] & 20.0 & 25.8 & 44.0 & 34.9 \\
MiF WFE [nm] & 2.6 & 2.2 & 4.2 & 22.3 \\
HiF WFE [nm] & 2.2 & - & 2.6 & 8.4 \\
Roughness [nm] & 0.5 & 0.2 & 0.9 & 2.0 \\
\hline
\end{tabular}

value of $69 \mathrm{~nm}$ rms including these six optics (focus and WFS errors subtracted). The main residual aberrations are astigmatism, third-order spherical aberration, and trefoil, which will be easily corrected by AO. This final result fully confirms individual interferometric measurements on each mirror. This extreme quality will allow the better contrast expected on the SPHERE instrument, once coupled with the HODM, the coronagraph, and the other subsystems.

\section{Conclusion}

We have demonstrated the potential of stress polishing based on Active Optics in the frame of the VLT SPHERE high-contrast instrument dedicated to direct imaging of exoplanets. This challenging scientific field requires extreme qualities for optical surfaces, especially aspherical ones. By avoiding the generation of structured mid- and high-spatial frequency surface errors, stress polishing ensures that the instrument will provide its best performances on-sky, once coupled with the XAO system and coronagraphs. A superpolishing method was applied to decrease the surface roughness down to a few angstroms, thanks to the simple spherical polishing with a full-sized pitch tool. Final results were estimated using the PSD obtained from interferometric measurements. A comparison with the PSD of the VLT UT3 primary mirror shows that the final contrast of the instrument will not be limited by the instrument's aspherical optics quality. The three TMs were delivered, installed, and aligned on the SPHERE bench, and measurements done in focus after six optics fully confirm the results obtained by individual interferometric tests. This is a first step towards more complex mirrors, such as off-axis parabolas, whose shape not only includes astigmatism but also focus, coma, and trefoil terms. We hope this achievement will bring exoplanet discoveries nearer.

Acknowledgements. SPHERE is an instrument designed and built by a consortium consisting of IPAG, MPIA, LAM, LESIA, Laboratoire Fizeau, INAF, Observatoire de Genève, ETH, NOVA, ONERA, and ASTRON in collaboration with ESO.

\section{References}

Baranne, A. 1996, A\&ASS, 119, 373

Beuzit, J. L., Feldt, M., Dohlen, K., et al. 2010, Proc. SPIE

Couder, A. 1940, CRAS Paris 210, 327

Dae Wook, K., \& Burge, J. H. 2010, Opt. Exp., 18, 2242

Fusco, T., Rousset, G., Sauvage, J. F., et al. 2006, Opt. Exp., 14, 7515

Give'on, A., Shaklan, S. B., \& Green, J. J. 2006, Proc. SPIE, 6288

Hinkley, S., Oppenheimer, B. R., \& Soummer, R. 2007, ApJ, 669, 642

Hugot, E., \& Lemaitre, G. R. 2008, Appl. Opt., 47, 1401

Hugot, E., Ferrari, M., El Hadi, K., et al. 2009, Appl. Opt., 48, 2932

Ingalls, A. G. 1937, Scientific American, Inc., New York

Jacob, S. D., Arrasmith, S. R., Kozhinova, I. A., et al. 1999, Am. Ceramic Soc. Bull., 78, 42

Leistner, A. J. 1993, Appl. Opt., 32, 3416

Leistner, A. J., Thwaite, E. G., Lesha, F., \& Bennett, J. M. 1992, Appl. Opt., 31, 1472

Lemaitre, G. R. 1972, Appl. Opt., 11, 1630

Lemaitre, G. R. 2009, Astronomical optics and elasticity - Active Optics methods, 130 (Berlin: Springer)

Lyot, B. 1939, MNRAS, 99, 580

Macintosh, B. 2005, Proc. SPIE 5903, 170

Macintosh, B. 2006, Proc. SPIE, 6272, 62720L

Marioge, J. P. 1984, J. Optics, 15, 119

Marois, C., Macintosh, B., Soummer, R., Poyneer, L., \& Bauman, B. 2008, Proc. SPIE, 7015

Mayor, M., \& Queloz, D. 1995, Nature, 378, 355

N'diaye, M., Dohlen, K., \& Cuevas, S. 2010, A\&A, 509, A8

Racine, R., Walker Gordon, A. H., Nadeau, D., et al. 1999, PASP, 111, 587

Roddier, F., \& Roddier, C. 1997, PASP, 109, 815

Rouan, D., Riaud, P., Boccaletti, A., Clénet, Y., \& Labeyrie, A. 2000, PASP, 112, 777

Schmidt, B. 1932, Mitt Hamburg Strenv., 7, 15

Soummer, R., Dohlen, K., \& Aime, C. 2003, ESA Pub. Div., 599

Soummer, R., Ferrari, A., Aime, C., \& Jolissaint, L. 2007, ApJ, 669, 642

Texereau, J. 1948, Société Astronomique de France

Timoshenko, S. P., \& Woinovsky-Krieger, S. 1959, Theory of plates and shells (McGraw-Hill Co.)

Vigan, A., Moutou, C., Langlois, M., et al. 2010, MNRAS, 407, 71

Walsh, C. J., Leistner, A. J., \& Oreb, B. F. 1999, Appl. Opt., 38, 4790

Weis, O. 1992, Appl. Opt., 31, 4355 